\title{
Intravesical Therapies in Non-muscle Invasive Bladder Tumors
}

\author{
- Serdar Geyik MD \\ Private Avrupa Hospital, Clinic of Urology, Adana, Turkey
}

\begin{abstract}
In the European Association of Urology (EAU) 2015 Guidelines for non-muscle invasive bladder tumors, maintenance Bacillus Calmette-Guérin (BCG) therapy is a grade A recommendation. In the intermediate-risk group, re-evaluation is recommended after 1-year full-dose treatment; in the highrisk group, full-dose BCG is recommended for 1-3 years. Intravesical BCG therapy fails in $40 \%$ patients in an average of 2 years. In these cases, there is no alternative treatment that is considered effective. In patients with failed BCG, comparison of BCG and gemcitabine showed less recurrence in the long term with gemcitabine while progression and toxicity were similar. Early radical cystectomy should be considered in non-muscle invasive bladder cancer patients with BCG-refractory T1G3 who have good performance status and low comorbidity. In T1 tumors, invade deeper than 3 mm and/or larger than $6 \mathrm{~mm}$ in diameter has been associated with a 100\% progression rate. BCG decreased recurrence more significantly in high-risk Ta and T1 tumors. In terms of progression of high-risk superficial bladder cancer, comparison of mitomycin C and BCG showed that BCG is superior if maintenance therapy is given. EAU guidelines recommend early bladder chemotherapy instillation (EBCl), in the low-to-intermediate risk group. There was no clear effect of $\mathrm{EBCl}$ in the intermediate- and high-risk group. $\mathrm{EBCl}$ alone reduces recurrence only in the low-risk group. However, adjuvant intravesical chemotherapy (AIVC) is recommended in the intermediate- and high-risk groups because it improves relapse-free survival. BCG and maintenance BCG therapy were found to be more effective than AIVC in reducing progression and preventing recurrence.
\end{abstract}

Keywords: Intravesical BCG, intravesical chemotherapy, early bladder chemotherapy instillation, non-muscle invasive bladder tumors

\section{Introduction}

Urothelial tumors are the fourth most common of all tumors (1). Bladder tumors account for $90-95 \%$ of the cases, while $5-10 \%$ originate in the upper urinary tract (2). At initial diagnosis, most cases (75-85\%) are non-muscle invasive bladder cancer (NMIBC). According to pathological T staging, $70 \%$ are classified as $\mathrm{Ta}, 20 \%$ as $\mathrm{T} 1$, and $10 \%$ as carcinoma in situ (CIS) (3). CIS is prone to muscle invasion (54\%) if not treated effectively. Recurrence, progression, and prognosis in NMIBC are predicted using nomograms and European Organization for Research and Treatment of Cancer (EORTC) and Spanish Urological Club for Oncological Treatment (CUETO) classification based on pathological examination of transurethral resection of the bladder (TUR-B) specimens. Adjuvant therapy decisions during follow-up of patients with NMIBC are made based on risk group stratification (low, intermediate, high) according to the clinical and pathological data. The EORTC classification is believed to require strengthening with new parameters due to several reasons such as the absence of additional pathological variants, lack of prognostic factors in the $\mathrm{T} 1$ tumor invasion subgroups, and lack of lymphovascular invasion criteria in histopathology (4). Muscle invasion occurs in about $30 \%$ of high-risk NMIBCs during follow-up. For high-grade T1 tumors, survival is as low as $34 \%$. This may be attributable to lower grade in initial pathology or the tumor having invasive characteristics (5). A study performed in 2011 showed that patients with invasive progression had worse prognosis than patients whose initial pathology was muscle invasive (3-year survival rate, $37 \%$ vs $67 \%$ ) (6). Previous studies suggested the need to define a "very-high-risk" subgroup within the high-risk NMIBC group in order to avoid the delay of correct treatment (7). As a result, the 2014 European Association of Urology (EAU) NMIBC guidelines included a highest-risk subgroup in addition to the low-, intermediate-, and high-risk groups and recommend early radical cystectomy (Table 1).

In cases with persistent T1 disease after secondary TUR-B, the progression rate for $\mathrm{T} 1 \mathrm{G} 3$ patients with 3 poor prognostic factors (age over 70 years, presence of $\mathrm{CIS}$, tumor larger than $3 \mathrm{~cm}$ ) was reported as $52 \%$. Progression rates of $40 \%$ were reported 
for female patients with $\mathrm{T} 1 \mathrm{G} 3$ or in the presence of prostatic $\mathrm{CIS}$. Strikingly, invasion deeper than $3 \mathrm{~mm}$ and/or greater than $6 \mathrm{~mm}$ in diameter was associated with 100\% progression in T1 patients (8). The standard follow-up and treatment of NMIBC is cystoscopy. In low-grade Ta disease, 3-year progression is rare (5\%), while recurrence rates are high (50-70\%). High-grade T1 disease shows substantially high rates of both progression (50\%) and recurrence (80\%) (9) (Table 2). In this study, we evaluated the clinical role and efficacy of intravesical treatments in NMIBC in light of the studies and reviews conducted to date.

\section{Bacillus Calmette-Guérin Therapy and Prognosis in Bladder Cancer}

Bacillus Calmette-Guérin (BCG) vaccine contains attenuated bacteria and its use within the bladder was first described by Morales in 1976 (10). It remains the most effective treatment method for the prevention of recurrence and progression in high-risk NMIBC. Application 2-4 weeks after TUR is recommended to prevent adverse effects. Urinary tract infections must be either excluded or treated before BCG therapy. The procedure should be postponed in cases of traumatic catheterization or other causes of disrupted tissue integrity. After BCG instillation, it should remain in the bladder for 1-2 hours (dwell time). Although this therapy has been used for more four decades, the mechanism of action is thought to be related to the immune response that develops due to local T-cell activation in the bladder mucosa. The local immune response induced by BCG involves both cellular and humoral immune mechanisms. Long-term follow-up of treated patients has revealed predominant increase in T cells and Th1type cytokines. Local inflammation induced by BCG is called granuloma and is known to play an important role in reducing tumor recurrence (11). The amount of bacteria in standard dose BCG solution should be 108 colony-forming units (CFU)/mL or higher. In their 2013 meta-analysis, Zhu et al. (12) determined that a below-standard BCG dose was associated with higher recurrence rate. However, CUETO previously showed that treatment with a one-third dose reduced recurrence and

Table 1. Very high-risk subgroup in the European Association of Urology 2014 Guidelines on non-muscle invasive bladder cancer

T1G3 with concurrent bladder CIS

T1G3 with concurrent prostatic urethral CIS

Multiple and/or large $(>3 \mathrm{~cm}) \mathrm{T} 1 \mathrm{G} 3$ tumors and/or recurrent T1G3

Presence of variant histology (especially micropapillary variant)

Lymphovascular invasion

CIS: Carsinoma in situ

Table 2. Analysis of 3-year recurrence and progression in lowgrade and high-grade T1 tumors $(9,36)$

\begin{tabular}{|l|l|l|}
\hline Tm pathology & $\begin{array}{l}\text { Recurrence } \\
\text { (3-year follow-up) }\end{array}$ & $\begin{array}{l}\text { Progression } \\
\text { (3-year follow-up) }\end{array}$ \\
\hline Low-grade $\mathrm{Ta}$ & $50-70 \%$ & $5 \%$ \\
\hline High-grade $\mathrm{T} 1$ & $80 \%$ & $50 \%$ \\
\hline
\end{tabular}

progression in selected intermediate-risk patients. Although the authors emphasized that full-dose BCG may not be necessary for effective treatment based on their results, their selection of moderate-risk patients for the study makes it difficult to draw a definitive conclusion on this issue (13). Treatment of ureteral and bladder cancer depends on cancer stage and grade. Considering that three-quarters of cases are NMIBC at diagnosis and that one-quarter exhibit progression, there is an undeniable need for additional treatment to prevent both recurrence and progression. BCG is still considered the most effective treatment in the prevention of recurrence and progression. However, careful patient selection is important due to its toxic effects. While intravesical BCG treatment is regarded as unnecessary in the low-risk group, it is strongly recommended for the high-risk group (Table 3). In the intermediate-risk group, the decision to administer intravesical BCG therapy is made based on an evaluation of the likelihood of recurrence and progression. In particular, CIS in addition to superficial bladder cancer should be managed as an invasive tumor. BCG therapy is regarded as first-line treatment for NMIBC patients with CIS (14). In another study it was determined that BCG with maintenance therapy should be the treatment of choice for intermediateand high-risk patients with CIS (15). Among the recent studies in the literature, a retrospective 2018 study by Yorozuya et al. (16) including 53 patients with variant histology (squamous or glandular differentiation) stands out. Their results support that in NMIBC, intravesical BCG therapy for variant histology has better prognosis in terms of progression and cancer-specific survival than other intravesical treatments (mitomycin $C$ and thiotepa) with no need for additional treatment.

\section{Bacillus Calmette-Guérin Therapy Doses (Induction and Maintenance)}

The currently accepted BCG induction protocol is that described by Morales et al. (10), in which BCG is instilled once a week for six weeks. Intravesical BCG therapy prevents not only prevents recurrence, but also progression. Therefore, it is especially recommended for the high-risk group. Unlike induction, there are no optimal treatment protocols for maintenance therapy. An EORTC study demonstrated the superiority of 3-year fulldose maintenance therapy to 1-year maintenance therapy in high-risk patients. In the same study, this effect was not observed in intermediate-risk patients; therefore, 1-year full-dose maintenance therapy is recommended for the intermediaterisk group. The use of different doses in that study showed no difference between one-third and full-dose maintenance BCG therapy in terms of toxicity (17). Maintenance therapy is a grade A recommendation in the 2015 EAU guidelines. Reevaluation after 1-year full-dose in the intermediate-risk group and continuation of full-dose maintenance BCG for 1-3 years in the high-risk group is recommended (18). Lamm et al.

Table 3. Spanish Urological Oncology Group low- and high-risk groups

\begin{tabular}{|l|l|}
\hline Low-risk group & Primary, Ta, solitary, $\mathrm{Tm}<3 \mathrm{~cm}, \mathrm{G} 1$, no CIS \\
\hline High-risk group & $\mathrm{T} 1, \mathrm{G} 3$, presence of CIS \\
\hline CIS: Carsinoma in situ \\
\hline
\end{tabular}


(19) analyzed the outcomes of a 6-week induction followed by 3-week treatment cycles performed at 3, 6, 12, 18, 24, 30, and 36 months. Particularly in CIS and selected Ta-T1 patients, maintenance intravesical BCG therapy was shown to approximately double the recurrence-free period.

\section{Adverse Effects of Bacillus Calmette-Guérin Therapy}

Local and systemic infections are adverse events that occur after BCG therapy. Hypersensitivity reactions (20) and adverse effects secondary to active infection (21) have been reported. The absolute and relative contraindications to BCG therapy must be known prior to treatment (Table 4) (22). The local adverse effects of intravesical BCG have been investigated in six different randomized studies. The main factors associated with adverse events were age over 70 years, immunosuppression, and urothelial damage. The most common local adverse effects included pollakiuria (71\%), cystitis (67\%), fever (25\%), and hematuria (23\%) (23). Lamm (24) reported the rate of severe complications as below $5 \%$ in their retrospective study. The most common adverse effect is fever (2.9\%), and antituberculosis treatment should be considered if it exceeds $39^{\circ} \mathrm{C}$ for more than 48 hours. Severe hematuria (1\%), granulomatous prostatitis $(0.9 \%)$, pneumonia, hepatitis, arthralgia $(<0.8 \%)$, and more rarely, epididymitis, ureteral obstruction, bladder contracture, and renal abscess have also been reported in the literature. The most common complication is BCG cystitis. It develops within 2-4 hours of treatment and usually resolves within 48 hours. The most feared adverse effect is systemic disease and sepsis. The development of systemic disease has been reported in the first 8-12 weeks. Diagnosis can only be made in the presence of granuloma in tissue biopsies. Infections secondary to BCG therapy may not always manifest with leukocytosis (25). Classical sepsis is very rare (26).

\section{Non-response to Bacillus Calmette-Guérin}

Intravesical BCG therapy fails in $40 \%$ of NMIBCs in an average of 2 years. In such cases, there is no alternative intravesical therapeutic agent considered completely effective. Progression is rare in patients with initial low-grade pathologic diagnosis. For NMIBC patients classified as high-risk, the probability of muscle invasion and progression must not be overlooked. The main factors in treatment failure are previous exposure to mycobacteria, inappropriate BCG dose, lack of expected cellular immune response in the individual, and therapies initiated based on missing or inaccurate staging (27). Early cystectomy is recommended after failed BCG therapy in T1G3 patients with good performance status and low comorbidity. Promising studies on the development of bladder-sparing intravesical therapies for patients with treatment failure are ongoing.

\begin{tabular}{|l|l|}
\hline \multicolumn{2}{|l|}{ Table 4. Absolute and relative contraindications for BCG therapy } \\
\hline $\begin{array}{l}\text { Absolute contraindications } \\
\text { for BCG }\end{array}$ & $\begin{array}{l}\text { Relative contraindications } \\
\text { for BCG }\end{array}$ \\
\hline - Immunosuppression & - Urinary tract infection \\
- History of BCG sepsis & - Liver disease \\
- Macroscopic hematuria & - History of tuberculosis \\
- Total incontinence & - Advanced age \\
- Early post-TUR & - Poor performance status \\
- Traumatic catheterization & \\
\hline BCG: Bacillus Calmette-Guérin, TUR: Transurethral resection \\
\hline
\end{tabular}

\section{Intravesical Chemotherapy in Non-muscle Invasive Bladder} Cancer

The addition of IVCT to TUR-B aims to eliminate residual tumor and prevent progression and recurrence (28). The superiority of any of the IVCT agents (mitomycin C, epirubicin, pirarubicin, and thiotepa) to one another has yet to be demonstrated (29). Choice of drug is based on cost, adverse effects, and practitioner experience. Mitomycin $C$ is the most frequently used agent. Its mechanism of action is the inhibition of DNA synthesis. It is an antimicrobial and anticancer agent obtained from Streptomyces species. In normal conditions, systemic absorption is limited due to its molecular weight (334 kD). However, it is not recommended for patients with mucosal damage, widespread resection, and radiotherapy (7). Although an optimum protocol has not been established, urine alkalinization followed by instillation of $40 \mathrm{mg} / 20 \mathrm{~mL}$ mitomycin $C$ and dwell time of 1 hour was recommended (30). When applied as $20 \mathrm{mg} / 50 \mathrm{~mL}$ dose, recurrence was reported to decrease from $57 \%$ to $17 \%$, and when used for $6-8$ weeks at higher doses $(40-80 \mathrm{mg})$, the proportion of high-grade bladder cancers that were recurrence-free at 2 years was reported as $75 \%$ (31). Applying mitomycin C with hyperthermia (41$44{ }^{\circ} \mathrm{C}$ ) was reported to increase treatment efficacy and survival. However, the use of this technique is limited due to increased cost associated with the special catheter necessary for hyperthermia, increased adverse effects of mitomycin $\mathrm{C}$ due to hyperthermia, and patient compliance problems (32). The most common effects associated with mitomycin C toxicity are chemical cystitis (40\%) (33) and allergic skin reactions (5-12\%) (34). In intravesical applications performed after TUR$B$, normal epithelialization of healing areas may be disrupted and replaced by dystrophic calcification, or extravasation from the bladder may cause necrosis, peritonitis, and pelvic pain (35). A 2003 review by Shelley et al. (36) including 6 studies compared BCG and mitomycin C treatments in $\mathrm{Ta}$ and $\mathrm{T} 1$ tumors. BCG resulted in a more pronounced reduction in the recurrence of high-risk Ta and T1 tumors. Local adverse effects (dysuria, pollakiuria, cystitis, hematuria) and systemic adverse effects (fever, fatigue) were in favor of BCG. A total of 108 patients were divided into passive mitomycin $\mathrm{C}$, electromotive mitomycin C, and BCG groups. Complete response rates at 6 months were $31-58 \%$ for passive and electromotive mitomycin C, respectively, and 64\% with BCG. Adverse effects were most frequent in the electromotive mitomycin $C$ group and least frequent in the group administered BCG only (37). Five studies with an average follow-up of 26 months compared BCG with and without maintenance therapy to mitomycin C in terms of progression of high-risk superficial bladder cancer. As a result, it was emphasized that BCG treatment is superior if maintenance therapy is provided (38). In another study, 212 T1 patients were divided into two groups, BCG only or sequential BCG and electromotive mitomycin C. Both groups underwent maintenance therapy. At the end of 88 months of follow-up, all parameters (recurrence, progression, and survival) were better in the group that received sequential treatment. Disease-free interval was 69 vs 21 months, recurrence rate was $42 \%$ vs $58 \%$, and disease-specific mortality rate was $6 \%$ vs $16 \%$. In terms of preventing progression, the difference in progression rates was 
noteworthy ( $16.2 \%$ with BCG, $9.3 \%$ with sequential treatment) (39). In their meta-analysis of the long-term outcomes of a total of 2820 patients in 9 studies, Malmström et al. (40) compared mitomycin C and BCG therapy. Over 4.4 years of follow-up, the recurrence rate was $43 \%$. There was no difference between BCG and mitomycin C in terms of time to first recurrence. If maintenance BCG was administered, recurrence was $32 \%$ lower compared to mitomycin C. A notable recent publication is a 2018 review by Chantada-Abal et al. (41) which evaluated the safety and efficacy of sequential intravesical BCG and mitomycin C in NMIBC. Their analysis revealed that sequential treatment does not cause more toxicity and leads to prolonged disease-free survival by reducing tumor progression more than BCG or mitomycin C monotherapy. They emphasized the need for further clinical research to enable the adoption of combination therapy into routine practice. Anthracyclines (valrubicin, epirubicin, doxorubicin): drugs in this group have lower systemic absorption and cause fewer systemic adverse effects due to their higher molecular weight. They exert their effects by inhibiting DNA topoisomerase 2 and protein synthesis, and via free radical formation and direct cytotoxicity on the cell membrane (42). Valrubicin is FDA-approved for BCG-refractory CIS patients who are ineligible for cystectomy. A complete response rate of $21 \%$ was reported in 90 BCGrefractory CIS patients. Adverse effects are local (symptoms of bladder irritation such as cystitis and hematuria) (43). Epirubicin has fewer adverse effects than doxorubicin but similar efficacy (31). Epirubicin is used at various doses between $20-100 \mathrm{mg}$, but is most frequently used at $50 \mathrm{mg} / 50 \mathrm{~mL}$ once a week for a total of 8 weeks. In a study by EORTC, comparison of single-dose $80 \mathrm{mg}$ epirubicin with TUR-B alone showed that epirubicin reduced recurrence by $12-15 \%$ (44). More recently, intravesical BCG and epirubicin therapies in patients with Ta and $\mathrm{T} 1$ bladder cancer were analyzed in terms of recurrence, progression by stage, mortality, distant metastases, and adverse effects. Five studies (549 patients treated with BCG and 562 with epirubicin) were included in the analysis. Recurrence rate was $51.4 \%$ in the epirubicin group and $35.5 \%$ in the BCG group. Differences between the two groups in terms of distant metastases and progression were not statistically significant. In 2 of the 5 studies analyzed, mortality and morbidity were equal in both groups. Local (cystitis, hematuria) and systemic toxicity (fever, fatigue) were more common in the BCG group (45). Gemcitabine acts by inhibiting cellular growth and inducing apoptosis. It has proven systemic efficacy in metastatic bladder cancer. While the results of its intravesical use are promising, more phase 3 studies are required to determine dosing and efficacy. In primary Ta-T1, patients who did not have CIS and did not receive additional treatment had comparable recurrence and progression rates while adverse effects were less common than with BCG (10\% vs $45 \%)$. In the high-risk NMIBC group, compared to BCG alone, gemcitabine therapy was associated with shorter time to recurrence ( 25.5 months vs 39.4 months) and higher recurrence rate (53\% vs $28 \%$ ). In BCG-refractory patients, comparison of BCG and gemcitabine showed that recurrence occurred later and less frequently $(52 \%$ to $87 \%$ ) with gemcitabine therapy. Progression and toxicity were similar (46). Doxatel is an antineoplastic agent that acts by disrupting the microtubular network during cell division. In
2013, 54 BCG non-responders were followed for an average of 39.1 months. Complete response was achieved in $59 \%$ of the patients. Recurrence-free survival was $40 \%$ in at 1 year and $25 \%$ at 3 years. Cystectomy was performed on $24 \%$ of the patients. The 5-year disease-free survival rate was reported as $85 \%$ (47). Thiotepa is an FDA-approved agent and was the first to be used intravesically in NMIBC. However, it caused severe adverse effects because it is readily absorbed through the bladder mucosa and passes into the systemic circulation due to its low molecular weight. It is hardly ever used today due to its systemic (myelosuppression and secondary leukemia in up to $54 \%$ ) and local (irritative urinary symptoms up to $70 \%$ ) adverse effects (48). Interferon alpha is an immunomodulatory and antiproliferative agent. In terms of recurrence, it is less effective compared with BCG and mitomycin C (49).

\section{Early Single-dose Intravesical Chemotherapy}

One of the mechanisms regarded as most important in tumor recurrence is the implantation of residual tumor cells in the bladder after TUR on the mucosal surface. The purpose of early single-dose IVCT is the chemoresection of free tumoral tissues. Technically, it is recommended within the first 24 hours (preferably within the first hours) after TUR-B (50). It is listed as a recommendation in the EAU guidelines for the treatment of lowto-intermediate risk bladder cancers. In a meta-analysis of 1476 patients by Sylvester et al. (51), it was shown that recurrence decreased by $11.7 \%$ with early single-dose IVCT. Independent of agent used, recurrence rates are reported as $65 \%$ in patients with multiple tumors and $36 \%$ in patients with solitary tumors. In a study evaluated based on the EORTC bladder cancer risk classification, it was shown that epirubicin treatment reduced recurrence by $28 \%$ in the low-risk group (score 0-2) but had no effect on recurrence in the high-risk group (score 3 or higher). It was emphasized that the best response to treatment was in cases with solitary tumors, and that the same response was not achieved in recurrent multifocal cases (52). Solsona et al. (53) reported that early single-dose IVCT only prevents recurrences occurring within 2 years after TUR-B, but did not prevent recurrences in the longer term. No clear effect of early single-dose IVCT could be demonstrated on progression, recurrence, and time to recurrence in the intermediate- and high-risk group. As a result, the EAU guidelines recommend early single-dose IVCT for low- to intermediate-risk patients with a single tumor focus (54). Choice of drug is based on past experience, accessibility, low cost, and fewer adverse effects. There are no drugs with definitively proven superiority. It is not recommended for wide and very deep resections due to the possibility of extravasation from the bladder and associated risk of systemic toxicity. Secondly, the most important hidden factor in treatment success may be a good pathological examination and obtaining a second confirmation from a different pathology department. Based on these analyses, intravesical approaches to NMIBC can be summarized as follows: early single-dose IVCT alone decreases recurrence only in the low-risk group, and adjuvant IVCT is recommended in the intermediate- and high-risk groups because it increases recurrence-free survival (55). Although the duration of adjuvant IVCT is controversial, less than a year is recommended (56). When TUR-B + IVCT 
was compared with TUR-B alone, the recurrence rate decreased by $13-14 \%$ with the addition of IVCT (57). BCG and BCG maintenance therapy were found to be more effective than IVCT in reducing progression and preventing recurrence (58).

\section{Ethics}

Peer-review: Externally peer-reviewed.

Financial Disclosure: The author declared that this study received no financial support.

\section{References}

1. Munoz JJ, Ellison LM. Upper tract urothelial neoplasms: incidence and survival during the last 2 decades. J Urol 2000;164:1523-1525.

2. Siegel R, Naishadham D, Jemal A. Cancer statistics, 2012. CA Cancer J Clin 2012;62:10-29.

3. Margulis V, Shariat SF, Matin SF, et al. Outcomes of radical nephroureterectomy: a series from the Upper Tract Urothelial Carcinoma Collaboration. Cancer 2009;115:1224-1233.

4. Lamm DL. Carcinoma of the bladder. Tex Med 1985;81:32-34.

5. Tanıdır Y, Tınay I. Kasa invaze olmayan mesane kanseri: çok yüksek riskli alt grupta tanı ve tedavi. Bull Urooncol 2015;14:50-54.

6. Van den Bosch S, Alfred Witjes J. Long-term cancer-specific survival in patients with high-risk, non-muscle-invasive bladder cancer and tumour progression: a systematic review. Eur Urol 2011;60:493-500.

7. Herr HW, Donat SM. A re-staging transurethral resection predicts early progression of superficial bladder cancer. BJU Int 2006;97:1194-1198.

8. Brimo F, Wu C, Zeizafoun N, et al. Prognostic factors in T1 bladder urothelial carcinoma: the value of recording millimetric depth of invasion, diameter of invasive carcinoma, and muscularis mucosa invasion. Hum Pathol 2013;44:95-102.

9. Lutzeyer W, Rübben H, Dahm H. Prognostic parameters in superficial bladder cancer: an analysis of 315 cases. J Urol 1982;127:250-252.

10. Morales A, Eidinger D, Bruce AW. Intracavitary Bacillus CalmetteGuerin in the treatment of superficial bladder tumors. J Urol 1976;116:180-183.

11. Kapoor R, Vijjan V, Singh P. Bacillus Calmette-Guérin in the management of superficial bladder cancer. Indian J Urol 2008;24:72-76.

12. Zhu S, Tang Y, Li K, et al. Optimal schedule of bacillus calmetteguerin for non-muscle-invasive bladder cancer: a meta-analysis of comparative studies. BMC Cancer 2013;13:332.

13. Martínez-Piñeiro JA, Solsona E, Flores N, Isorna S. Improving the safety of BCG immunotherapy by dose reduction. Cooperative Group CUETO. Eur Urol 1995;27(Suppl 1):13-18.

14. Hall MC, Chang SS, Dalbagni G, et al. Guideline for the management of nonmuscle invasive bladder cancer (stages Ta, T1, and Tis): 2007 update. J Urol 2007; 178:2314-2330.

15. Sylvester RJ, van der MEIJDEN AP, Lamm DL. Intravesical bacillus Calmette-Guerin reduces the risk of progression in patients with superficial bladder cancer: a meta-analysis of the published results of randomized clinical trials. J Urol 2002;168:1964-1970.

16. Yorozuya W, Nishiyama N, Shindo T, et al. Bacillus Calmette-Guérin may have clinical benefit for glandular or squamous differentiation in non-muscle invasive bladder cancer patients: retrospective multicenter study. Jpn J Clin Oncol 2018;48:661-666.

17. Oddens J, Brausi $M$, Sylvester R, Bono A, et al. Final results of an EORTC-GU cancers group randomized study of maintenance bacillus Calmette-Guérin in intermediate- and high-risk Ta, T1 papillary carcinoma of the urinary bladder: one-third dose versus full dose and 1 year versus 3 years of maintenance. Eur Urol 2013;63:462-472.

18. Babjuk M, Böhle A, Burger M, et al. EAU Guidelines on Non-Muscleinvasive Urothelial Carcinoma of the Bladder: Update 2016. Eur Urol 2017;71:447-461.
19. Lamm DL, Blumenstein BA, Crissman JD, et al. Maintenance bacillus Calmette-Guerin immunotherapy for recurrent TA, T1 and carcinoma in situ transitional cell carcinoma of the bladder: a randomized Southwest Oncology Group Study. J Urol 2000;163:1124-1129.

20. Elkabani M, Greene JN, Vincent AL, et al. Disseminated Mycobacterium bovis after intravesicular bacillus calmette-Gu rin treatments for bladder cancer. Cancer Control 2000;7:476-481.

21. McParland C, Cotton DJ, Gowda KS, et al. Miliary Mycobacterium bovis induced by intravesical bacille Calmette-Guérin immunotherapy. Am Rev Respir Dis 1992;146:1330-1333.

22. Jones JS, Larchian WA. Non-muscle-invasive bladder cancer ( $\mathrm{Ta}, \mathrm{T} 1$ and CIS). In: Wein AJ, Kavoussi LR, eds. Cambell-Walsh Urology. 10th ed. Philadelphia: PA; 2012. p. 2335-2354.

23. Shelley MD, Court JB, Kynaston H, et al. Intravesical bacillus CalmetteGuerin versus mitomycin C for Ta and T1 bladder cancer. Cochrane Database Syst Rev 2003:CD003231.

24. Lamm DL. Efficacy and safety of bacille Calmette-Guérin immunotherapy in superficial bladder cancer. Clin Infect Dis 2000;31(Suppl 3):86-90.

25. Soylu A, Ince AT, Polat $H$, et al. Peritoneal tuberculosis and granulomatous hepatitis secondary to treatment of bladder cancer with Bacillus Calmette-Guérin. Ann Clin Microbiol Antimicrob 2009;8:12.

26. Diner EK, Verghese M. Interstitial pneumonitis secondary to intravesical bacillus calmette-guerin for carcinoma in-situ of the bladder. Int Braz J Urol 2004;30:400-402.

27. Witjes JA. Management of BCG failures in superficial bladder cancer: a review. Eur Urol 2006;49:790-797.

28. O'Donnell MA. Practical applications of intravesical chemotherapy and immunotherapy in high-risk patients with superficial bladder cancer. Urol Clin North Am 2005;32:121-131.

29. Sylvester RJ, Oosterlinck W, van der Meijden AP. A single immediate postoperative instillation of chemotherapy decreases the risk of recurrence in patients with stage $\mathrm{Ta} T 1$ bladder cancer: a metaanalysis of published results of randomized clinical trials. J Urol 2004; 171:2186-2190.

30. Au JL, Badalament RA, Wientjes MG, et al. Methods to improve efficacy of intravesical mitomycin C: results of a randomized phase III trial. J Natl Cancer Inst 2001;93:597-604.

31. Gofrit ON, Shapiro A, Pode D, et al. Combined local bladder hyperthermia and intravesical chemotherapy for the treatment of high-grade superficial bladder cancer. Urology 2004;63:466-471.

32. Colombo R, Salonia A, Leib Z, et al. Long-term outcomes of a randomized controlled trial comparing thermochemotherapy with mitomycin- $\mathrm{C}$ alone as adjuvant treatment for non-muscle-invasive bladder cancer (NMIBC). BJU Int 2011;107:912-918.

33. Böhle A, Jocham D, Bock PR. Intravesical bacillus Calmette-Guerin versus mitomycin $C$ for superficial bladder cancer: a formal metaanalysis of comparative studies on recurrence and toxicity. J Urol 2003;169:90-95.

34. Colver GB, Inglis JA, McVittie E, et al. Dermatitis due to intravesical mitomycin C: a delayed-type hypersensitivity reaction? $\mathrm{Br}$ J Dermatol 1990;122:217-224.

35. Koya MP, Simon MA, Soloway MS. Complications of intravesical therapy for urothelial cancer of the bladder. J Urol 2006;175:2004-2010.

36. Shelley MD, Court JB, Kynaston H, et al. Intravesical bacillus CalmetteGuerin versus mitomycin C for Ta and T1 bladder cancer. Cochrane Database Syst Rev 2003:CD003231.

37. Di Stasi SM, Giannantoni A, Stephen RL, et al. Intravesical electromotive mitomycin $C$ versus passive transport mitomycin $C$ for high risk superficial bladder cancer: a prospective randomized study. J Urol 2003;170:777-782.

38. Böhle A, Bock PR. Intravesical bacille Calmette-Guérin versus mitomycin $C$ in superficial bladder cancer: formal metaanalysis of comparative studies on tumor progression. Urology 2004;63:682-686. 
39. Di Stasi SM, Giannantoni A, Giurioli A, et al. Sequential BCG and electromotive mitomycin versus BCG alone for high-risk superficial bladder cancer: a randomised controlled trial. Lancet Oncol 2006;7:43-51.

40. Malmström PU, Sylvester RJ, Crawford DE, et al. An individual patient data meta-analysis of the long-term outcome of randomised studies comparing intravesical mitomycin $C$ versus bacillus Calmette-Guérin for non-muscle-invasive bladder cancer. Eur Urol 2009;56:247-256.

41. Chantada-Abal V, Chantada-Tirado C, Lamas-Díaz L, et al. Sequential treatment with Mitomycin $C$ and BCG in non muscle invasive bladder cancer. Arch Esp Urol 2018;71:453-457.

42. Duque JL, Loughlin KR. An overview of the treatment of superficial bladder cancer. Intravesical chemotherapy. Urol Clin North Am 2000;27:125-135.

43. Steinberg G, Bahnson R, Brosman S, et al. Efficacy and safety of valrubicin for the treatment of Bacillus Calmette-Guerin refractory carcinoma in situ of the bladder. The Valrubicin Study Group. J Urol 2000;163:761-767.

44. Oosterlinck W, Kurth $\mathrm{KH}$, Schröder F, et al. A prospective European Organization for Research and Treatment of Cancer Genitourinary Group randomized trial comparing transurethral resection followed by a single intravesical instillation of epirubicin or water in single stage Ta, T1 papillary carcinoma of the bladder. J Urol 1993;149:749-752.

45. Shang PF, Kwong J, Wang ZP, et al. Intravesical Bacillus CalmetteGuérin versus epirubicin for Ta and T1 bladder cancer. Cochrane Database Syst Rev 2011:CD006885.

46. Jones G, Cleves A, Wilt T], et al. Intravesical gemcitabine for nonmuscle invasive bladder cancer. Cochrane Database Syst Rev 2012;1:CD009294.

47. Barlow LJ, McKiernan JM, Benson MC. Long-term survival outcomes with intravesical docetaxel for recurrent nonmuscle invasive bladder cancer after previous bacillus Calmette-Guérin therapy. J Urol 2013;189:834-839.

48. Hollister D Jr, Coleman M. Hematologic effects of intravesicular thiotepa therapy for bladder carcinoma. JAMA 1980;244:2065-2067.

49. Malmström PU. A randomized comparative dose-ranging study of interferon-alpha and mitomycin- $\mathrm{C}$ as an internal control in primary or recurrent superficial transitional cell carcinoma of the bladder. BJU Int 2002;89:681-686.

50. Soloway MS, Masters S. Urothelial susceptibility to tumor cell implantation: influence of cauterization. Cancer 1980;46:1158-1163.

51. Sylvester RJ, Oosterlinck W, van der Meijden AP. A single immediate postoperative instillation of chemotherapy decreases the risk of recurrence in patients with stage Ta T1 bladder cancer: a metaanalysis of published results of randomized clinical trials. J Urol 2004; 171:2186-2190.

52. Gudjónsson S, Adell L, Merdasa F, et al. Should all patients with nonmuscle-invasive bladder cancer receive early intravesical chemotherapy after transurethral resection? The results of a prospective randomised multicentre study. Eur Urol 2009;55:773-780.

53. Solsona E, Iborra I, Ricós JV, et al. Effectiveness of a single immediate mitomycin $C$ instillation in patients with low risk superficial bladder cancer: short and long-term followup. J Urol1999;161:1120-1123.

54. Dobruch J, Herr H. Should all patients receive single chemotherapeutic agent instillation after bladder tumour resection? BJU Int 2009; 104:170-174.

55. Tolley DA, Parmar MK, Grigor KM, et al. The effect of intravesical mitomycin $C$ on recurrence of newly diagnosed superficial bladder cancer: a further report with 7 years of follow up. J Urol 1996; 155:1233-1238

56. Sylvester RJ, Oosterlinck W, Witjes JA. The schedule and duration of intravesical chemotherapy in patients with non-muscle-invasive bladder cancer: a systematic review of the published results of randomized clinical trials. Eur Urol 2008;53:709-719.

57. Elmamoun MH, Christmas TJ, Woodhouse CR. Destruction of the bladder by single dose Mitomycin $C$ for low-stage transitional cell carcinoma (TCC)--avoidance, recognition, management and consent. BJU Int 2014;113:34-38.

58. Böhle A, Bock PR. Intravesical bacille Calmette-Guérin versus mitomycin $C$ in superficial bladder cancer: formal metaanalysis of comparative studies on tumor progression. Urology 2004;63:682-686. 